\title{
SIDA et maternité
}

Patrice Cohen et Suniti Solomon (éds), AIDS and Maternity in India, Pondicherry, French Institute of Pondicherry, 2004, 279 p.

Frédéric Bourdier

\section{(2) OpenEdition}

12 Journals

Édition électronique

URL : http://journals.openedition.org/transcontinentales/1474

DOI : 10.4000/transcontinentales. 1474

ISBN : 978-2-8218-1408-0

ISSN : $1775-397 X$

\section{Éditeur}

Editions de la maison des sciences de l'homme

\section{Édition imprimée}

Date de publication : 30 juin 2006

Pagination : 183-183

ISBN : 2200-92169-1

ISSN : $1950-1684$

\section{Référence électronique}

Frédéric Bourdier, «SIDA et maternité », Transcontinentales [En ligne], 2 | 2006, document 17, mis en ligne le 15 décembre 2012, consulté le 25 septembre 2020. URL : http://journals.openedition.org/ transcontinentales/1474; DOI : https://doi.org/10.4000/transcontinentales.1474

Ce document a été généré automatiquement le 25 septembre 2020.

Tous droits réservés 


\section{SIDA et maternité}

Patrice Cohen et Suniti Solomon (éds), AIDS and Maternity in India, Pondicherry, French Institute of Pondicherry, 2004, 279 p.

\section{Frédéric Bourdier}

\section{RÉFÉRENCE}

PATRICE Cohen ET SUNITI Solomon (ÉDS)

AIDS and Maternity in India, Pondicherry, French Institute of Pondicherry, 2004, 279 p.

1 Treize auteurs abordent la problématique de la transmission maternelle du VIH en Inde et retracent les grandes lignes de la stratégie nationale de lutte contre le sida avec une mise en perspective de la politique engagée au Brésil et en Afrique subsaharienne.

Des contributions proposent des angles d'approches précis : modes vernaculaires de sustentation et d'allaitement, représentations et pratiques liées à l'accouchement, retombées de la violence et de la discrimination féminine envers la santé reproductive. D'autres écrits fournissent un cadre de présentation générale censé faire le point sur des programmes régionaux, principalement en Tamil Nadu. Certains de ces textes pèchent par une rhétorique peu informative qui ne reflète guère ce qui se passe concrètement : c'est le cas de la présentation consacrée à la prévention, qui offre une vision idéalisée du programme national, et de celle du programme UNICEF dans le Sud du pays, qui relève - tout au moins jusqu'en 2004 - plus d'une intention que d'une implantation concertée.

3 Parallèlement à l'intervention publique, qui peine à se doter d'une certaine efficacité tant épidémiologique que sociale et mobilisatrice, et reste à l'état de projet, l'ouvrage se concentre sur quelques expériences pragmatiques réalisées par le secteur non gouvernemental. Deux exemples montrent comment des priorités identifiées par des acteurs de terrain parviennent à être systématisées pour se tourner en actions durables. YRG care, une des premières ONG indiennes à s'être penchée sur les soins pour les personnes séropositives, ainsi que SIAAP, qui impulsa en pionnier les techniques de conseils au dépistage modulables en fonction des contextes locaux, témoignent de la 
pugnacité de certaines initiatives ayant émergé en dehors de toute formulation gouvernementale, allant parfois à l'encontre de ce que l'État considérait comme primordial dans les années 1990.

4 Au-delà de la description des actions intentées, certains auteurs font preuve de discernement et montrent à quel point les orientations en matière de santé publique, notamment celles qui touchent à la prévention de la transmission mère/enfant, sont alimentées pas des choix et restent des constructions sociales. Un système de santé est un système politique, et la gestion de l'épidémie à VIH témoigne de ce jeu complexe d'interactions au sein duquel la question médicale stricto sensu doit composer avec des forces sociales qui la dépassent et l'enserrent.

5 Si des analyses de cet ouvrage collectif, issu d'un séminaire organisé en 2002 à Pondichéry, méritent d'être approfondies, il convient de rappeler que les propos échangés lors de la rencontre relèvent de débats qui partaient de faits encore peu discutés. Le présent livre a donc le mérite de lancer des perspectives de recherche innovantes dont les décideurs et les développeurs continuent à sous-estimer la pertinence. Autant de considérations qu'il serait judicieux de prendre en compte dans les politiques à venir.

\section{AUTEURS}

\section{FRÉDÉRIC BOURDIER}

Institut de recherche pour le développement (IRD) 